\title{
Identification of co-expression gene networks controlling rice blast disease during an incompatible reaction
}

R. Bevitori ${ }^{1}$, S. Sircar ${ }^{2}$, R.N. de Mello ${ }^{1}$, R.C. Togawa ${ }^{3}$ MV C B. Côrtes 1 T.S. Oliveira ${ }^{4}$, M.F. Grossi-de-Sá ${ }^{3}$ and N. Parekh ${ }^{2}$

${ }^{1}$ Embrapa Arroz e Feijão, Santo Antônio de Goiás, GO, Brasil

${ }^{2}$ Center for Computational Natural Sciences and Bioinformatics, International Institute of Information Technology, Hyderabad, India

${ }^{3}$ Embrapa Recursos Genéticos e Biotecnologia, Brasília, DF, Brasil

${ }^{4}$ Instituto de Biologia, Unicamp, Campinas-SP, Brasil

Corresponding author: R. Bevitori

E-mail: rosangela.bevitori@embrapa.br

Genet. Mol. Res. 19 (3): gmr18579

Received February 11, 2020

Accepted July 03, 2020

Published July 31, 2020

DOI http://dx.doi.org/10.4238/gmr18579

ABSTRACT. Rice blast disease is a major threat to rice production worldwide; the causative pathogenic fungus Magnaporthe oryzae induces rice (Oryza sativa) plants to undergo molecular changes that help them to circumvent this fungal attack. Transcriptome studies have demonstrated that many genes are involved in the defense response of rice to $M$. oryzae, but most of these studies focused on the screening of differentially expressed genes and the studies did not investigate the interactions among genes. We examined the interaction of rice and $M$. oryzae in a network context. Two near-isogenic lines were profiled at different time-points. Using transcriptome data obtained from an RNASeq analysis, a network based on the relationships among genes was developed through weighted gene co-expression network analysis. The analysis of degree centrality identified numerous hub genes and potential key regulators that control the rice response, providing new insights into the molecular network underlying the resistance of rice to M. oryzae infection. Additionally, a protein-protein interaction network was derived to identify complexes that might physically interact. For example, complexes of OsbHLH148/OsJAZ, OsMYB4 and some components of the phenylpropanoid pathway, as well as MYB/bHLH 
and NB-LRR/OsWRKYs were identified, suggesting possible roles in regulating $M$. oryzae infection. The combination of in silico data with transcription factor binding indicates that OsbZIP45 may serve as a driver of complex gene expression changes that result in resistance to rice blast disease, and can thus act as an integrator of multiple signals and as a coordinator of diverse cellular pathways to control the defense responses.

Key words: Oryza sativa; Magnaporthe oryzae; Transcriptome; WGCNA; Weighted gene co-expression network

\section{INTRODUCTION}

Rice (Oryza sativa) blast disease places a major constraint on global rice production. Depending on the inoculum pressure, crop growth stage at the time of infection, cultivar susceptibility, environmental conditions and management system, the fungus Magnaporthe oryzae in rice fields can cause losses in rice production of around $30 \%$ (Nalley et al., 2016). Although rice cultivars exhibit different reactions to blast disease, very few cultivars show resistance, and this resistance frequently deteriorates within a few years due to high variability in the pathogen population (Koizumi, 2007).

Magnaporthe oryzae develops a very complex interaction network with rice. This hemibiotrophic pathogen first establishes a biotrophic phase (Talbot et al., 2003). During this phase, the host's immune system is actively suppressed, and this suppression allows the spread of invasive hyphae to throughout the infected plant tissue. The fungus then switches to a necrotrophic lifestyle in which toxins are secreted by the pathogen to induce host cell death. The interactions within this pathosystem involve bidirectional recognition. On the one hand, plants need to sense or recognize the foreign molecules delivered by the pathogen to activate plant innate immunity, and this process is denoted the pathogen-associated molecular pattern (PAMP)-triggered immunity (PTI). On the other hand, for successful infection, the pathogen needs to identify special target proteins that can disrupt the immune system-effector-triggered immunity (ETI) of plants (Hong and Ziding, 2016). During PTI and ETI, plants trigger a diverse array of immune responses, such as reactive oxygen species (ROS) generation, cellular $\mathrm{Ca}^{2+}$ spikes, MAP kinase (MAPK) activation, phytohormone production, and transcriptional reprogramming, to increase the defense response to $M$. oryzae infection. Changes in gene expression and transcriptional reprogramming are major features of plant immunity that are governed by transcription factors (TFs) and coregulatory proteins associated with discrete transcriptional complexes (Moore et al., 2011). TF and their associated co-factors are integrated within signaling pathways and, upon receptor activation and signal initiation, they decode this information in various manners to induce diverse transcriptional changes (Tsuda and Somssich, 2015). (Tsuda and Somssich, 2015).

Several transcriptome studies have demonstrated that a large number of genes are involved in the defense response of rice to M. oryzae (Wei et al., (2013), but most of these studies focused on the screening of individual differentially expressed genes and ignored the high interconnection among genes. In recent years, global co-expression networks have been constructed to study human diseases (Voigt et al., 2017) and the response of plant to biotic and abiotic stresses (Sircar and Parekh, 20015; Onaga et al., 2017) and these 
networks provided an overview of gene interrelationships at the systems level. It is generally accepted that most genes work together in pathways and complexes to form a network of molecular interactions that determine the biological processes required for survival in response to environmental changes (Whitfield et al., 2002). Thus, in this study, we obtained RNA sequencing (RNA-Seq) data from two near-isogenic lines (NILs) of rice at 4, 8, 12 and 24 hours after infection (hai) and mock controls (no infection) and performed a transcriptome analysis of these data to identify a set of candidate defense genes associated with $M$. oryzae during an incompatible reaction (successful plant defense). A co-expression network was constructed using the $\mathrm{R}$ package based on a Weighted Gene Co-expression Network Analysis (WGCNA) (Langfelder and Horvath, 2008) to identify significant gene modules and hub genes associated with resistance to $M$. oryzae. We generated a proteinprotein interaction (PPI) network to identify which specific genes/transcription factors (TFs) interacted with each other to elucidate their functional role in rice defense against $M$. oryzae. An additional analysis using a transcriptional regulatory network approach was performed to investigate whether TFs induced at 4 hai would drive the gene expression changes observed in rice at 24 hai. Together, these analyses identified many hub genes and potential key regulators that control the response of rice and, thereby providing new insights into the molecular networks underlying rice resistance to $M$. oryzae infection.

\section{MATERIAL AND METHODS}

\section{Plant material and blast inoculation}

The aim of this study was to explore the interaction of rice and M. oryzae during an incompatible reaction in a network context. Two near-isogenic lines (NILs), IRBL5-M (resistant line carrying Pi5) and IRBLI-F5 (susceptible line carrying Pii) were used in this study. They share the same genetic background as Lijiangxin-tuan-heigu (LTH), a cultivar bred by International Rice Research Institute (IRRI) (Tsunematsu et al., 2000). Three-weekold rice plants were inoculated by spraying a conidial suspension from the fungal isolate 9881 at $3 \times 10^{5}$ spores $/ \mathrm{mL}$ (Fillipi and Prabhu, 2001). The isolate 9881 is a monospore isolate deposited at the Embrapa Arroz e Feijão Collection of Multifunctional Microorganisms at http://www.cnpaf.embrapa.br/transferencia/informacoestecnicas /colecao_fitopatogenos/ ind x.php). Each sample consisted of 10 rice leaf blades from 10 different plantlets, and three biological replicates were obtained for each NIL and condition (mock vs blast-inoculated). The plants were maintained in a humidity chamber at $25^{\circ} \mathrm{C}$. At $0,4,8,12$, and 24 hai, the third leaf of each plantlet was collected and immediately frozen in liquid nitrogen and stored at $-80^{\circ} \mathrm{C}$ until use. After seven days, the macroscopic phenotypes (necrosis and lesions) on rice leaves during the compatible interaction (successful infection) were observed and served as a control for the quality of the inoculation procedure.

\section{Sample preparation}

Total RNA was extracted using the RNeasy kit (Qiagen, Germany) and treated with an RNase-Free DNase Set (Qiagen, Germany), according to the manufacturer's instructions. The RNA quality, library construction and size were assayed using a 2100 Bioanalyzer 
system (Agilent, USA). The RNA from the three biological replicates of each treatment was pooled and used for sequencing. The libraries were synthesized using the TruSeq RNA Sample Preparation v2 kit (Illumina, USA). The samples were run in the HiSeq2500 system using two lanes and raw sequences of 51-bp were obtained. Two Fastq files were generated from each sample (one from each lane). The raw reads obtained using the HiSeq2500 analysis were filtered and further checked for sequence contaminants using the FastQC application at http://www.bioinformatics.babraham.ac.uk/projects/fastqc.

\section{Sequences processing and analysis}

FastQC was used to estimate the raw reads and assess their quality (Anders, 2010). Trimming of reads was carried out using Trimmomatic (Bolger et al., 2010). Reads containing contaminant primer/adapters were removed. Additionally, reads with leading and trailing base calls below a PHRED score of 3 were removed. After these steps, reads which were less than 36 bases long were removed. Each sample was mapped to O. sativa_204 (version 9.0) from the MSU Rice Genome Annotation Project (Kawahara et al., 2013) and redundancies were removed using the Picard suite of tools (http://broadinstitute.github.io/picard/). Alignment was performed using the metrics module of SAMtools (Li et al., 2009). The quality of the alignment was analyzed using Qualimap (Garcia-Alcalde et al., 2012). The read counts were estimated using HTSeq using "gene" as the "feature-type" (Anders et al, 2015). Normalization of the read counts and differential expression analysis were performed using DESeq (Anders et al., 2010). For a given genotype, differentially expressed genes (DEGs) at each time-point were identified based on a 2 -fold change (FC) (ratio of the number of transcripts in the inoculated sample to the number of transcripts in the mock control $\geq 2$ and a padj-value $<0.05$ ).

\section{Expression analysis by reverse transcription quantitative real-time PCR (RT- qPCR)}

The expression levels of 12 genes identified as DEGs were validated by RT-qPCR using the Power SYBR® Green kit (Applied Biosystems, USA), according to the manufacturer's instructions. The RNA samples used for the qRT-PCR assays were the same as those used for the RNA-Seq experiments. Total RNA was used for the synthesis of cDNA using the SuperScript ${ }^{\circledR}$ III First-Strand Synthesis System (Thermo Fisher), according to the manufacturer's instructions. The primers used in the assay were designed with GenScript at https://www.genscript.com/tools/pcr-primers-designer. The amplification assays were performed using a 7500 system (Applied Biosystems, USA). The rice ubiquitin 5 gene UBQ5 (GenBank accession AK061988.1) was used as the internal reference in the RT-qPCR assay. Three technical replicates were run, and the relative gene expression was calculated using the $2-^{\Delta \Delta \mathrm{Ct}}$ method. The values were expressed as the means \pm standard deviations (SDs). The corresponding gene-specific primers used in the gene expression analysis are listed in Table Supplementary 1).

\section{Co-expression network analysis}

Using the raw read counts obtained with HTSeq, a common expression matrix for the two NILs and the time-points $(4,8,12$ and 24 hai) as well as the mock control was 
constructed. The expression matrix was normalized using the relative log expression (RLE) normalization method in the DESeq $\mathrm{R}$ package. The similarity between gene pairs was computed using a signed Pearson's correlation matrix that was scaled to power $\beta=26$ based on the approximate scale free-topology criterion. The blockwiseModules function was used for the hierarchical clustering of genes based on the dynamic tree cut approach (Langfelder and Horvath, 2008) with a maximum block size of 20,600 and a minimum module size of 200. To identify genes with high connectivity (i.e., hubs), the topologically significant genes in the stress-responsive modules were determined using the WGCNA function intramodularConnectivity (Langfelder et al., 2013), and values for $k_{\text {Within }}$ (a measure of the connectivity (or degree) of a gene within a given co-expressed module) were obtained. The top $20 \%$ of the highly connected genes were selected as hub genes and further analyzed in detail. Functional classification of the differentially expressed transcripts was performed using the MapMan hierarchical ontology program (version 3.6) (Thimm et al., 2004). The top $20 \%$ of the highly connected genes were selected as hub genes and further analyzed for functional classification of the differentially expressed transcripts were performed using the MapMan hierarchical ontology program (version 3.6).

\section{Protein-protein interactions and regulatory networks}

The interactions among TFs and genes co-expressed at 24 hai were extracted from String DB (confidence score of 0.5) (Szklarczyk et al., 2015). In the results, each node represents a protein and the edges connect proteins that bind to each other. To predict the regulatory links between TFs and their target genes, we used TF2Network (Kulkarni et al., 2018). The Arabidopsis orthologs for 224 up-regulated genes in the important modules (salmon and magenta) in the resistant NIL were retrieved and submitted to TF2Network. The candidate TF regulators for a set of co-expressed or functionally related genes based on enriched TF-binding sites (TFBS) were thus obtained.

\section{RESULTS AND DISCUSSION}

\section{RNA-sequencing and validation of RNA-Seq data by RT-qPCR}

We performed a transcriptome analysis using RNA-Seq data from two NILs, IRBL5-M and IRBLI-F5 at 0, 4, 8, 12 and 24 hai. A total of 77,273,110 high-quality reads (average length $=51 \mathrm{bp}$ ) were generated with an Illumina HiSeq 2500 sequencer (NCBI SRA Sample accession: PRJNA546131). Each sample and biological condition was represented by an average of 9.66 million reads and $\sim 85 \%$ of the reads in each sample mapped to the rice genome (data not shown). To confirm the gene expression patterns identified from the RNA-seq data, the transcript levels of 12 randomly selected genes were examined by RT-qPCR (quantitative real time polymerase chain reaction). All the gene expression patterns obtained were found to be consistent with the RNA-seq data (Table Supplementary 1).

\section{Construction of gene co-expression networks}

The WGCNA R package was used to construct a 'signed' co-expression network consisting of the normalized read counts (20,521 genes). The resulting 16 co-expressed 
modules ranged in size from 3,055 (turquoise) to 334 (light cyan) genes, and 1,503 genes remained unclustered, as shown in the dendrogram presented in Figure 1.

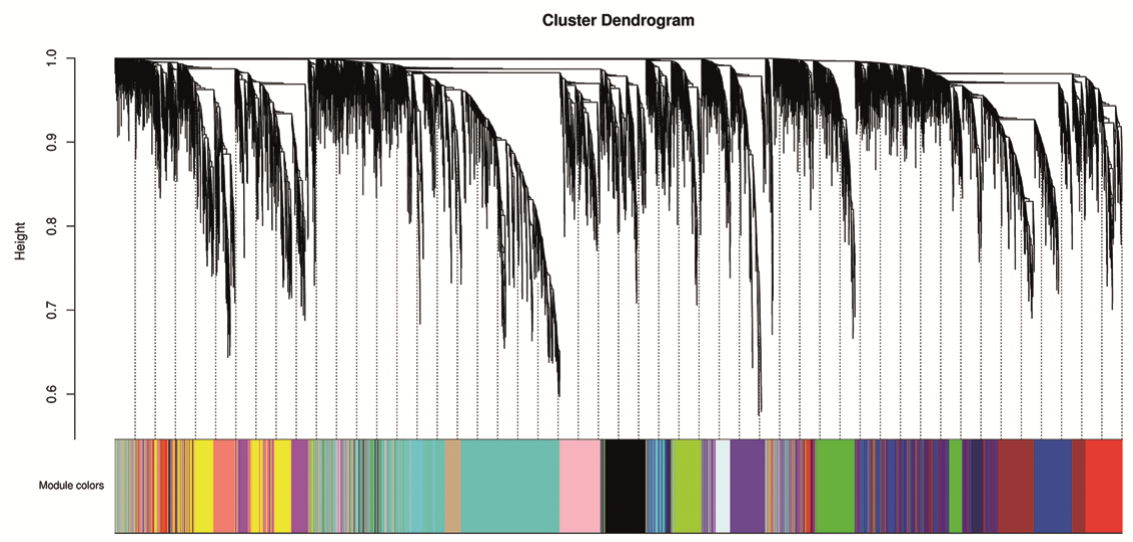

Figure 1. Dendrogram of all expressed gene sets was constructed using Topological Overlap Measure (TOM) matrix as a measure of dissimilarity for the detection of co-expression clusters. The gene clusters (modules) are represented by the colors.

Modules are defined as clusters of highly interconnected genes, and the genes within the same cluster have high correlation coefficients. In WGCNA, the term "coexpression" refers to the similarity among gene expression profiles under a given experimental condition (Langfelder and Horvath, 2008), and gene co-expression network illustrates the correlation between genes and can thus be used to represent the complexity of a cellular transcription network (Aoki et al., 2007). To identify stress-responsive modules unique or common to the resistant and susceptible NILs, the DEGs at each time-point and in each genotype were identified based on fold change (FC) of at least 2 and padj-value < 0.05. Those DEGs that were found to have these criteria were considered statistically significant. The DEGs computed for each of the time-points in the two NILs) were mapped to the 16 modules (Table Supplementary 2). In this table, modules exhibiting $\geq 10 \%$ of DEGs were considered to be enriched with up/down regulated genes. Notably, the salmon, magenta, yellow; brown, red and midnight-blue modules were enriched with up-regulated genes, whereas the black, light-cyan, pink, tan and turquoise modules comprised genes that were mainly down-regulated at the various points.

Notably, the salmon module contained a large percentage of DEGs across all timepoints in both NILs, which indicated its importance in disease resistance. Furthermore, in the resistant NIL, the percentage of DEGs in the salmon module increased from $4(10.1 \%)$ to 24 hai $(54.3 \%)$, whereas the reverse trend was observed for the susceptible NIL $(57.8 \%$ at 4 to $17.3 \%$ at 24 hai), which clearly indicated its role in providing resistance to infection. The yellow module was also identified as another important module because it contained genes that were up-regulated in both NILs at 4 and 8 hai and genes that were up-regulated in the resistant NIL at 24 hai (24R). From the Table Supplementary 2, we also observed that the analysis of the magenta module showed that a considerable fraction of its genes $(23.46 \%)$ was up-regulated at 4 hai in the resistant NIL, but not in the susceptible NIL $(2.3 \%)$, which suggested that the DEGs in the magenta module are specific to the resistant 
NIL. The genes in the brown, red, blue, and midnight-blue modules were up-regulated in a time-specific manner.

Among the down-regulated modules, the turquoise and black modules contained a large percentage of down-regulation genes in both NILs (Table Supplementary 2). For example, in the tan module, the fraction of DEGs observed at early time-points (4 and 8 hai) was comparable in both NILs, and the numbers decreased at later time-points (12 and 24 hai). In the pink module, DEGs in the resistant NIL were observed only at 8 hai, whereas a larger number of DEGs was observed in the susceptible NIL at 8, 12 and 24 hai. These findings clearly indicated the role of these DEGs in shutting down numerous processes in response to infection in the susceptible NIL.

\section{Identification of modules correlated with traits}

Based on the correlation of the module eigengene (a representative measure of the gene expression profiles of a module) with the sample trait (NILs + time-points), we identified co-expressed module genes associated with particular treatments (Figure 2).

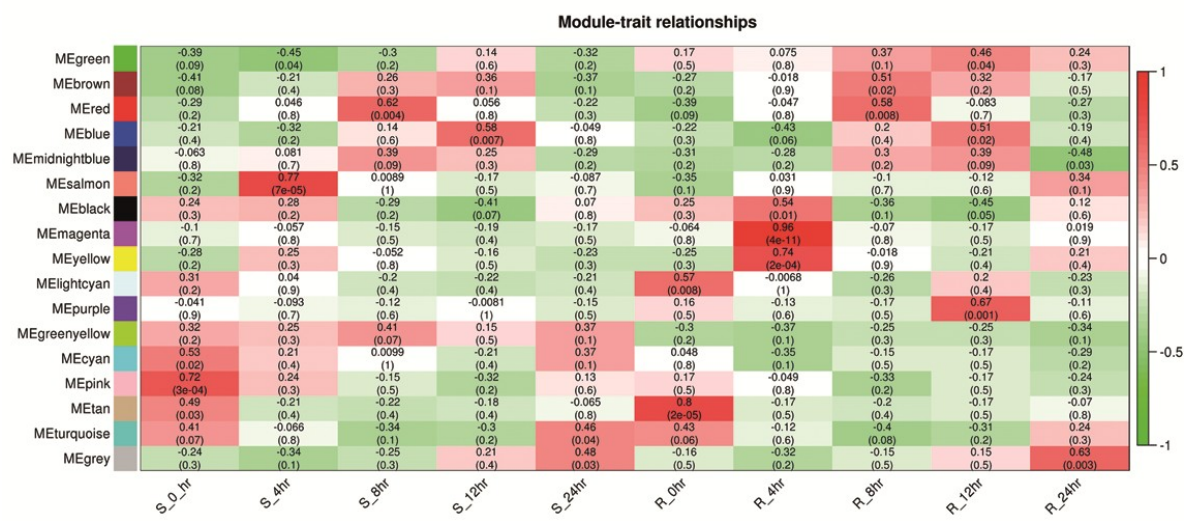

Figure 2. Pearson correlation coefficient of the module eigengene with the sample trait (genotype+time-point). Each cell reports the Pearson correlation between the module eigengene (rows) and the time-points (columns) with the corresponding $p$-values in parentheses. The red color represents a strong positive correlation whereas the green color indicates a strong negative correlation.

Based on the analysis of the expression pattern of genes at various time-points in the susceptible NIL, the strongest correlation $(r=0.77)$ was found for the salmon module at the earliest time-point (4 hai), followed by the red module $(\mathrm{r}=0.62)$ at 8 hai and the blue module $(\mathrm{r}=0.58)$ at 12 hai. Similarly, the gene expression pattern in the resistant NIL indicated that the magenta $(\mathrm{r}=0.96)$, yellow $(\mathrm{r}=0.74)$ and black $(\mathrm{r}=0.54)$ modules were positively correlated with the sample trait at the earliest time-point (4 hai). It has been suggested that 24 hai is a critical point for pathogen invasion. At the early stages of infection, specifically before and during 24 hours after pathogen inoculation, rice activates defense responses against $M$. oryzae (Bagnaresi et al., 2008). At 24 hai, rice blast fungi initiate appressorium penetration to reach the interior of rice leaves, and thus, this timepoint is considered the most suitable for extensive transcriptome analysis. Hence, the salmon module was selected for detailed analysis because it exhibited the highest 
correlation at 24 hai in the resistant NIL (24R). In addition, the turquoise module contained down-regulation genes in both NILs at 8 and 12 hai. Based on a systematic analysis of the distribution of DEGs across the modules and the correlation of the module eigengene with the trait, we selected the magenta and salmon modules for further analysis with the aim of identifying time-specific functional roles in the rice response to $M$. oryzae infection. The modules exhibiting contrasting representation of DEGs in the two NILs might be useful for obtaining an improved understanding of the biological processes associated with these treatments. At 4 and 24 hai, most fungal spores have only just germinated ( 4 hai) and have just begun to penetrate into rice epidermal cells, respectively (Ribot et al., 2008).

\section{Identification of co-expressed genes in the magenta module at 4 hai}

The magenta module presents a unique genotype-specific response in the resistant NIL. As shown in Table Supplemetary 2, 23.5\% of the genes in this module were up-regulated at 4 hai (4R) in the resistant NIL whereas only $3 \%$ of the genes were upregulated in the susceptible NIL at 4-hai (4S). This finding clearly indicates the probable role of the DEGs of this module in the early stress response in the resistant NIL. Representative genes among the most up-regulated DEGs at 4R (magenta module) are listed in Table 1.

In $4 \mathrm{R}$, the genes involved in the response to signaling were highly expressed and included TKL_IRAK_DUF26-lc.14, the leucine-rich repeat receptor protein kinase EXS precursor, and a Ras-related protein, with 3.1-, 5.5- and 4.6-fold changes, respectively. The highly expressed genes involved in response to secondary metabolites included the genes encoding leucoanthocyanidin dioxygenase, which showed a 6.52fold increase in expression. Genes encoding AMP-binding domain-containing protein and flavonol synthase/flavanone 3-hydroxylase exhibited more than 3-fold increases in expression in $4 \mathrm{R}$ during the incompatible interaction (successful plant defense), which indicated that rice might also utilize secondary metabolites to suppress rice blast fungal infection.

Our analyses also identified several genes involved in biotic stress, for example, the xylanase inhibitor protein 1 precursor CHIT16, a chitinase family protein precursor that is involved in the perception of chitin fragments during the first step in the plant defense response to fungal pathogens, showed a 4.2 -fold increase in expression (Table 1). Several genes related to lipid metabolism were transcriptionally activated in response to $M$. oryzae infection (e.g., acyl-desaturase showed a 6-fold increase in expression), and these results indicated that rice might also use this metabolism to suppress rice blast fungal infection. In addition, up-regulated genes involved in posttranslational modifications, serine/threonine protein kinase and calcium/calmodulindependent protein kinase (CAMK) activity, and abscisic acid (ABA) signaling were also identified. A significant feature of the magenta module is that metabolic processes corresponding to almost all the phytohormones that were reported in the biotic and abiotic stress response were captured in $4 \mathrm{R}$ and $4 \mathrm{~S}$ (Figures $3 \mathrm{~A}$ and $3 \mathrm{~B}$, respectively). These genes include, for example, genes involved in auxin signaling, the ABA 
signaling pathway, ethylene biosynthesis, brassinosteroid synthesis/degradation and signaling, and jasmonic acid (JA) biosynthesis. These genes were significantly regulated only in $4 \mathrm{R}$ (Table 1 ). These genes were significantly regulated only in $4 \mathrm{R}$ (Table 1).

Table 1. Genes of the magenta module showing significant up-regulation at 4 hours after infection in the resistant near-isogenic lines.

\begin{tabular}{|c|c|c|}
\hline Gene ID $^{\mathrm{a}}$ & RGAP Annotations $^{\mathrm{b}}$ & FC_ $4 \mathbf{R}^{\mathrm{c}}$ \\
\hline \multicolumn{3}{|c|}{ Secondary Metabolism } \\
\hline LOC Os03g18030 & leucoanthocyanidin dioxygenase & 6.5 \\
\hline LOC_Os01g67540 & AMP-binding domain containing protein & 5.0 \\
\hline LOC_Os05g03640 & flavonol synthase/flavanone 3-hydroxylase & 3.5 \\
\hline \multicolumn{3}{|l|}{ Stress.biotic } \\
\hline LOC Os06g24990 & xylanase inhibitor protein 1 precursor & 4.3 \\
\hline $\mathrm{LOC}^{-} \mathrm{Os} 03 \mathrm{~g} 04060$ & CHIT16 - Chitinase family protein precursor & 4.2 \\
\hline $\mathrm{LOC}^{-} \mathrm{Os} 07 \mathrm{~g} 17250$ & disease resistance RPP13-like protein 1 & 3.0 \\
\hline \multicolumn{3}{|l|}{ Lipid metabolism } \\
\hline LOC_Os03g53010 & acyl-desaturase, chloroplast precursor & 6.4 \\
\hline LOC_Os03g18010 & phospholipase $\mathrm{C}$ & 3.1 \\
\hline $\mathrm{LOC}^{-} \mathrm{Os} 10 \mathrm{~g} 31950$ & 3-ketoacyl-CoA thiolase, peroxisomal precursor & 2.4 \\
\hline \multicolumn{3}{|l|}{ Signāing } \\
\hline LOC_Os04g52780 & leucine-rich repeat receptor protein kinase EXS precursor & 5.5 \\
\hline LOC_Os07g35340 & $\begin{array}{l}\text { TKL_IRAK_DUF26-lc.14 - DUF26 kinases have homology to DUF26 } \\
\text { containing loci }\end{array}$ & 5.4 \\
\hline LOC_Os01g62950 & ras-related protein & 4.5 \\
\hline \multicolumn{3}{|c|}{ Hormone metabolism } \\
\hline LOC Os09g26780 & zinc-finger protein & 3.2 \\
\hline $\mathrm{LOC}^{-} \mathrm{Os} 03 \mathrm{~g} 49380$ & lipoxygenase & 3.1 \\
\hline $\mathrm{LOC}^{-} \mathrm{Os} 05 \mathrm{~g} 38290$ & protein phosphatase 2 & 2.3 \\
\hline $\mathrm{LOC}^{-}$Os $03 \mathrm{~g} 44380$ & 9-cis-epoxycarotenoid dioxygenase 1 , chloroplast precursor & 2.9 \\
\hline LOC_Os05g46350 & IQ calmodulin-binding motif domain containing protein & 5.0 \\
\hline $\mathrm{LOC}^{-} \mathrm{Os} 06 \mathrm{~g} 48950$ & auxin response factor 19 & 2.4 \\
\hline $\mathrm{LOC}^{-}$Os11g07950 & expressed protein & 3.5 \\
\hline LOC_Os02g55560 & protein phosphatase $2 \mathrm{C}$ & 3.3 \\
\hline $\mathrm{LOC}^{-} \mathrm{Os} 01 \mathrm{~g} 56810$ & cytokinin dehydrogenase precursor & 4.1 \\
\hline $\mathrm{LOC}^{-}$Os01g64790 & AP2 domain containing protein & 4.0 \\
\hline LOC_Os $12 \mathrm{~g} 07030$ & expressed protein & 6.3 \\
\hline \multicolumn{3}{|l|}{ Cell wall } \\
\hline LOC Os10g35460 & COBRA & 5.6 \\
\hline \multicolumn{3}{|l|}{ Cytochrome P450 } \\
\hline LOC Os12g39310 & cytochrome P450 & 6.3 \\
\hline LOC_Os01g63930 & cytochrome P450 & 2.5 \\
\hline \multicolumn{3}{|c|}{ Protein.post-translational modification } \\
\hline LOC_Os01g66860 & serine/threonine protein kinase & 4.5 \\
\hline LOC_Os $02 \mathrm{~g} 34600$ & $\begin{array}{l}\text { CAMK_CAMK_like.13 - CAMK includes calcium/calmodulin depedent } \\
\text { protein kinases }\end{array}$ & 3.3 \\
\hline LOC_Os07g35260 & $\begin{array}{l}\text { TKL_IRAK_DUF26-lc.9 - DUF26 kinases have homology to DUF26 } \\
\text { containing loci }\end{array}$ & 2.5 \\
\hline LOC_Os01g54480 & serine/threonine protein kinase & 1.3 \\
\hline LOC Os07g 36590 & serine/threonine-protein kinase receptor precursor & 2.3 \\
\hline \multicolumn{3}{|l|}{ Redox state } \\
\hline LOC_Os01g03914 & cation efflux family protein & 1.5 \\
\hline $\mathrm{LOC}^{-}$Os11g37890 & NAD dependent epimerase/dehydratase family protein & 1.0 \\
\hline $\mathrm{LOC}^{-} \mathrm{Os} 01 \mathrm{~g} 74450$ & aquaporin protein & 1.2 \\
\hline $\mathrm{LOC}^{-} \mathrm{Os} 02 \mathrm{~g} 45760$ & chitin-inducible gibberellin-responsive protein 2 & 2.2 \\
\hline $\mathrm{LOC}^{-} \mathrm{Os} 07 \mathrm{~g} 47990$ & peroxidase precursor & 3.6 \\
\hline
\end{tabular}



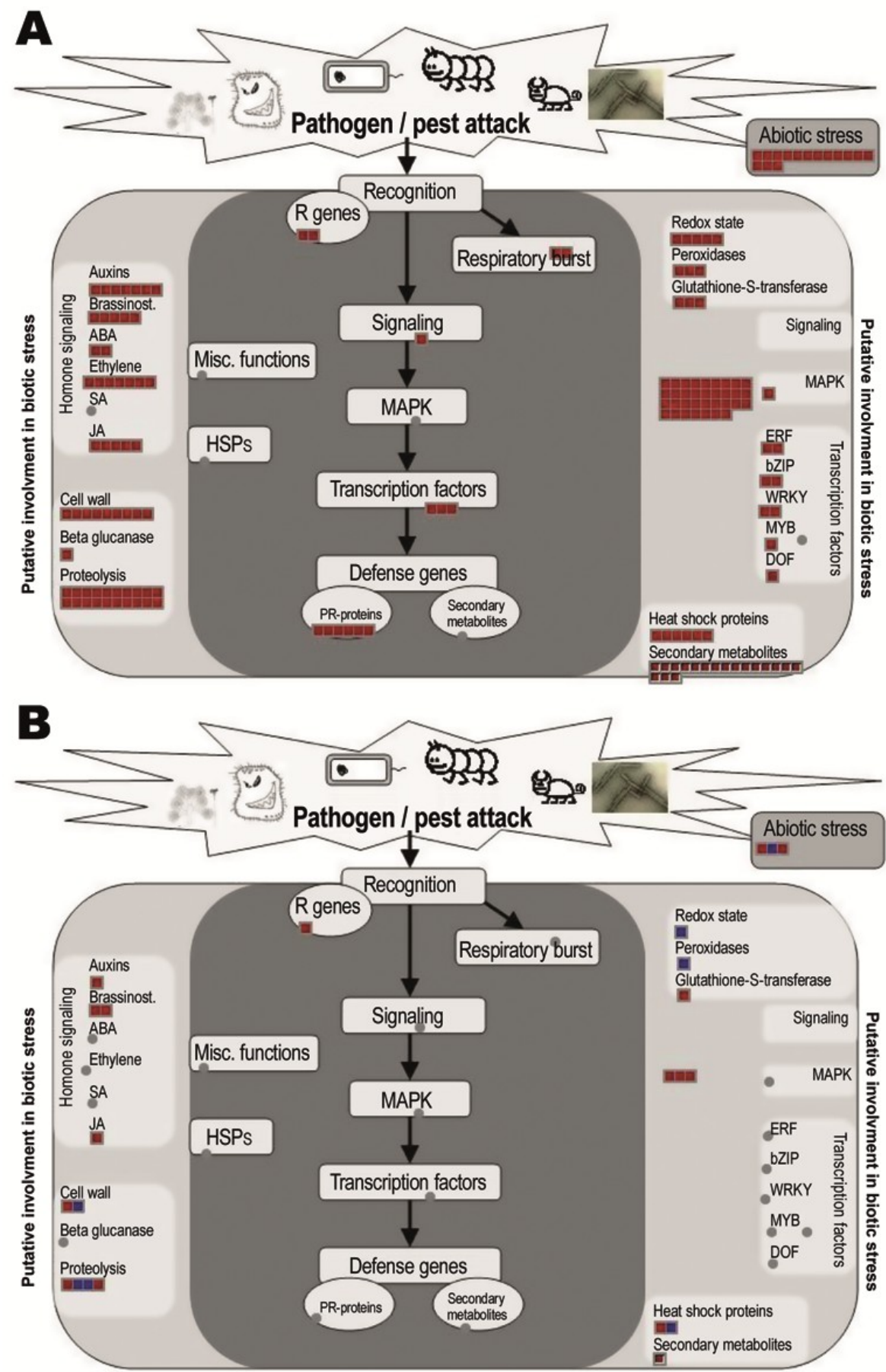

Figure 3. Overview of significant differentially expressed genes in the magenta module involved in the biotic stress pathway in MapMan. A) resistant near-isogenic lines at 4 hours after infection (hai). B) susceptible NIL at 4 hai. The red color shows up-regulated genes, and the blue color represents down-regulation genes. 


\section{Identification of hub genes at $\mathbf{4}$ hai in the magenta module}

To analyze whether the DEGs were also well connected with other genes, we performed a centrality-based analysis of the top 20\% (196) high-degree (hub) genes in the magenta module. Numerous studies have shown that highly expressed genes/proteins tend to be essential for organism function (Barabasi et al., 2004). Among the top 20\% of hub genes in the magenta module, 136 genes were up-regulated in $4 \mathrm{R}$, whereas only ten genes were up-regulated in 4S. The top 20 hub genes are listed in Table 2. In particular, several genes exhibiting high expression levels were also found to be associated with high-degree centrality (hubs), such as dehydrogenase, lipoxygenase ( $L O X)$, 3-ketoacyl-CoA thiolase $(A C C A)$, the AP2 domain-containing protein WRKY11, and acyl desaturase.

Table 2. List of the top 20 hub differentially expressed genes identified in the magenta module.

\begin{tabular}{|c|c|c|c|}
\hline Gene ID $^{a}$ & RGAP $^{\text {b }}$ annotation & $F C^{c} \_$R & $\mathbf{k}_{\text {Within }}^{\mathbf{d}}$ \\
\hline LOC_Os05g49760 & dehydrogenase & 2.63 & 92.36 \\
\hline LOC_Os03g49380 & lipoxygenase & 3.10 & 91.61 \\
\hline LOC_Os10g31950 & 3-ketoacyl-CoA thiolase, peroxisomal precursor & 2.15 & 90.88 \\
\hline LOC_Os05g49770 & CTP synthase & 2.62 & 88.68 \\
\hline LOC_Os01g43650 & WRKY11 & 3.35 & 82.59 \\
\hline LOC_Os06g11090 & CXE carboxylesterase & 2.42 & 82.21 \\
\hline LOC_Os $12 \mathrm{~g} 07030$ & expressed protein & 6.27 & 80.91 \\
\hline LOC_Os05g46350 & $\begin{array}{l}\text { IQ calmodulin-binding motif domain containing } \\
\text { protein }\end{array}$ & 5.00 & 79.80 \\
\hline LOC_Os03g50960 & $\begin{array}{l}\text { LTPL1 } 18 \text { - Protease inhibitor/seed storage/LTP } \\
\text { family protein precursor }\end{array}$ & 3.04 & 79.41 \\
\hline LOC_Os04g57550 & $\begin{array}{l}\text { amine oxidase, flavin-containing, domain } \\
\text { containing protein }\end{array}$ & 2.57 & 79.30 \\
\hline LOC_Os05g29990 & $\begin{array}{l}\text { NAD dependent epimerase/dehydratase family } \\
\text { domain containing protein }\end{array}$ & 2.01 & 78.73 \\
\hline LOC_Os01g64790 & AP2 domain containing protein & 4.02 & 77.88 \\
\hline LOC_Os08g04110 & transporter, putative & 1.64 & 77.32 \\
\hline LOC_Os03g53010 & acyl-desaturase, chloroplast precursor & 6.39 & 79.77 \\
\hline LOC_Os08g04630 & $\begin{array}{l}\text { external NADH-ubiquinone oxidoreductase } 1 \text {, } \\
\text { mitochondrial precursor }\end{array}$ & 3.37 & 75.86 \\
\hline LOC_Os04g47620 & protein kinase APK1B, chloroplast precursor & 0.0 & 74.68 \\
\hline LOC_Os03g50160 & plastocyanin-like domain containing protein & 2.18 & 73.85 \\
\hline LOC_Os03g62790 & Pirin & 1.39 & 73.18 \\
\hline LOC_Os07g17330 & B12D protein & 2.38 & 73.13 \\
\hline LOC_Os09g38320 & phytoene synthase, chloroplast precurso & 1.78 & 73.12 \\
\hline
\end{tabular}

\section{Identification of co-expressed up-regulated genes at 24 hai in the salmon module}

The salmon module is another important module because it contains the highest percentage of up-regulated genes among all the co-expressed modules (Table Supplementary 3). Interestingly, the number of DEGs decreased from 4 to 24 hai in the susceptible NIL, whereas the reverse trend was observed in the resistant NIL . Additionally, the expression of the most up-regulated genes increased in the resistant NIL over time, with a peak at 24 hai. A large number of genes (94) involved in post-translational modifications 
and signaling were up-regulated (Table Supplementary 3), which suggested that signal perception was extensively activated in the resistant NIL at 24 hai (FC 1.23-6.33). Some examples of these genes are the cell wall-associated kinases (WAKs). OsWAK20 and some other WAKs bear a calcium-binding domain, and their expression in the resistant NIL contributed to the up-regulation of CAMKs in this NIL.

Genes encoding receptor-like cytoplasmatic kinases (RLCKs), such as lectin receptor kinases (FC 7.06) were also up-regulated in 24R (Table S3). Some lectin receptor kinases are reportedly involved in plant resistance to pathogens. For example, a previous study demonstrated that the rice gene $P i-d 2$ encoding the lectin-like receptor kinase provides resistance against the fungal pathogen M. oryzae (Chen et al., 2006). In our study, these genes did not show significant changes in expression in the susceptible NIL, which suggested that the possible the rice lectin-like receptor kinase might be involved in the defense response against $M$. oryzae challenge. Other genes that were involved in signaling and post-translational modification showed a $\geq 2$-fold increase in expression in the $24 \mathrm{R}$ (Table Supplementary 3). These included OsCDPK7, OsCPK4, OsMSR2/OsCML31, OsCML16, OsCPK15 and OsCPK20, which are all known to be involved in biotic and abiotic stresses (Bundo et al., 2016). Similarly, a leucine-rich repeat family protein also showed increased expression in the $24 \mathrm{R}$ (FC 2.78) compared with the $24 \mathrm{~S}$ (FC 0.77). In our analysis, several genes related to secondary metabolism were found to be transcriptionally activated in response to $M$. oryzae infection. These genes involved in phenylpropanoid biosynthesis and the lignin pathways included the phenylalanine ammonia-lyase gene (PAL) and the AMP-binding domain containing protein, which is similar to 4-coumarate CoA ligase 2 (4CL2) in Arabidopsis. The rice OSPAL gene family consists of nine members (OSPAL 1-9), and most of them are associated with disease resistance (Duan et al, 2014). For example, a mutant of OsPAL4 was found to increase the rice susceptibility to Rhizoctonia. solani, Xanthomonas oryzae, and M. oryzae (Tonnessen et al., 2015), whereas knockout mutant OsPal6 displayed increase susceptibility to M. oryzae (Duan et al., 2014). In our analysis, OsPAL3 was found to be transcriptionally activated in response to $M$. oryzae infection, showing increased expression in the $24 \mathrm{R}$ (FC 2.92) compared with the $24 \mathrm{~S}$ (FC -0.46) (Table Supplementary 3). However, its relation to rice resistance to M. oryzae remains to be experimentally elucidated.

Several genes associated with hormone metabolism were also identified in this module (Table Supplementary 3). Among these, genes associated with ethylene (ET), brassinosteroid (BR), and auxin metabolism showed the greatest up-regulation due to their crucial roles during plant-pathogen interactions. The ethylene signaling and response pathway includes ethylene response factors (ERFs), which belong to the APETALA2/ERF TF family. The ERF104 gene exhibited $\sim 5$-fold differential expression, but its function in the biotic stress response has not been characterized. Abiotic and biotic stress-associated TFs, e.g., OsNAC95 (FC 2.51) and WRKYs (FC 2.18 to 6.47) (Table Supplementary 3), were also observed to be up-regulated. The defense-related TFs, such as WRKY, NAC and AP2/ERF found in our study have mostly been induced in response to pathogens infection. However, exogenous application MoHrip1, a secreted protein elicitor isolated from M. oryzae, activated the expression of these set of TFs without the fungus inoculation of rice plants (Lv et al., (2016). In this study, the combination of these TFs and the expression of several defense-related genes was considered to enhance resistance to rice blast fungus. 
In addition to hormone-related functions, genes encoding pathogenesis-related (PR) proteins, such as thaumatin and pathogenesis-related protein (PR6), were also observed to be upregulated. Other resistance genes included disease resistance-like protein (RPP13-like) and nucleotide binding-ARC/leucine-rich repeat (NB-ARC/LRR), which exhibited the highest gene expression levels in the 24R (FC 7.54). It is interesting to highlight that the resistant NILs used in this study, IRBL5-M, carries the Pi5 gene and Pi5-mediated resistance to M. oryzae requires the presence of two adjacently located NBS-ARC/LRR genes, named Pi5-1 and Pi5-2 (Lee at al., (2009). In our study, network analysis (Table Supplementary 4) identified NB-ARC/LRR proteins that interact with several genes, including WRKY24, WRKY 70, receptor-like protein kinases, harpin-induced protein 1 domain containing protein (stress), aspartic proteinase nepenthesin precursor, CAMK_CAMK_like.12 - CAMK includes calcium/calmodulin dependent protein kinases, tyrosine protein kinase domain containing protein, inactive receptor kinase At1g27190 precursor, and BRASSINOSTEROID INSENSITIVE 1 precursor. Then, we speculate that NB-ARC/LRR may modulate post-transcriptional reprograming in activating processes involved in biotic stress after $M$. oryzae infection including signaling, receptor-like kinases, and regulation by transcription factors.

\section{Protein-protein interaction at the early stage of infection (24 hai)}

Co-expressed modules comprise genes that exhibit similar response to an external stress. Thus, based on the concept of guilt-by-association, they are likely to be part of related biological processes and may share the same cellular components. Incorporating known proteinprotein interactions among these genes help in identifying more robust and smaller clusters, some of which may represent protein complexes or sub-pathways in the network. Thus, to elucidate the functional roles of these genes, their interactions were extracted from the String database (confidence score of 0.5), and the results identified the 249 interactions between 72 genes (Figure 4 and Table Supplementary 4).

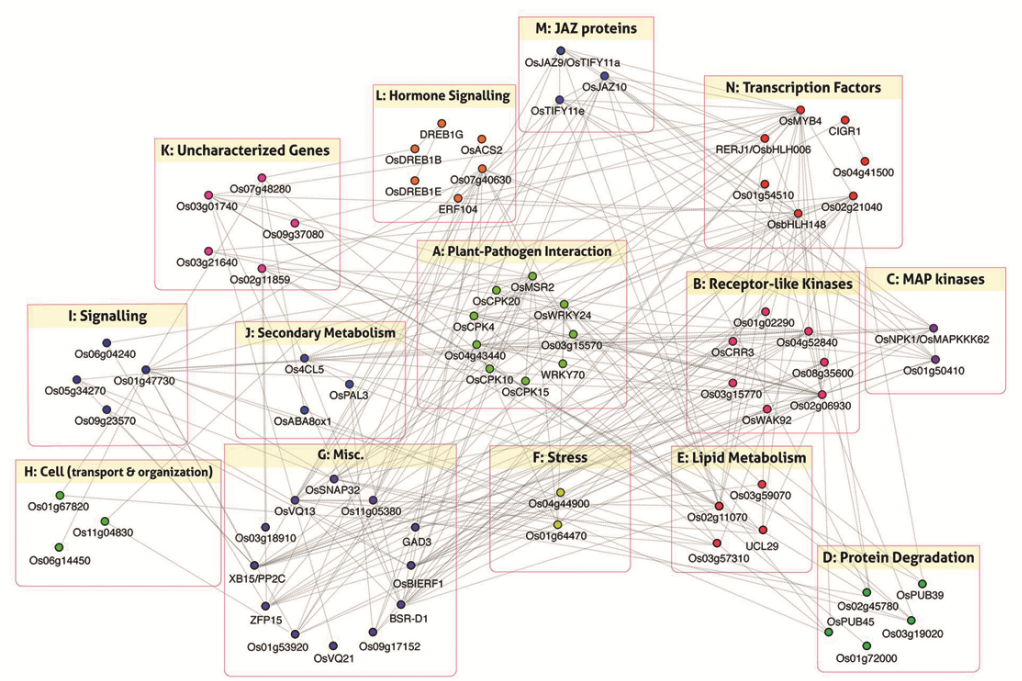

Figure 4. Protein-protein interaction network of high degree genes at 24 hours after infection in the salmon module. The clusters were based on KEGG and MapMan ontologies. 
Based on both MapMan and KEGG pathway ontologies, the genes were manually clustered into nine subcategories and several interactions were found to have a total score above 0.9, which provided high confidence to these interactions (Table Supplementary 4). In Figure 4, the cluster names are provided along with the gene names (MSU-LOC IDs). The nine genes were located at the center of the network, and were found to be directly involved in "Plant-Pathogen Interaction," as demonstrated by the KEGG analysis (Cluster A). Several interactions in the network were derived from this analysis and most of them were associated with both biotic and abiotic stresses. It is interesting to highlight that several TFs interact with other TFs, such as bHLH148 with MYB24 (score of 0.73) (Table Supplementary 4). A recent study showed that MYB cooperates with BHLH proteins in regulatory networks that control phenylpropanoid biosynthetic pathways and epidermal cell differentiation and patterning in Arabidopsis (Ramegowda et al., 2017). Here, we observed a network of regulatory interactions among plant defense and lipid, and secondary mechanisms that notably integrated with the phenylpropanoid pathway (Table Supplementary 4).

\section{Regulatory network for rice resistance to M. oryzae at 4 and 24 hai}

The construction of co-expressed modules using WGCNA yielded disjoint clusters. Consequently, the set of genes up-regulated in the magenta module at 4 hai, and those in the salmon module do not share any common gene or TF (Table Supplementary 5). Hence, we hypothesize that the up-regulation of co-expressed DEGs in the magenta module that are exclusively observed in $4 \mathrm{R}$ might play a role in the initial stimulation after pathogen invasion, which suggests a crucial purpose for acquiring $M$. oryzae resistance and conditioning the plants for enhanced responses against this pathogen. These genes might also activate the expression of other genes, many of which are harbored in the salmon module during appressorium penetration (24 hai), and thereby, priming the resistant NIL for enhanced survival.

To further investigate the regulatory role of the genes clustered in the magenta module, we extracted these set of genes in the magenta (228) and salmon (354) modules that were up-regulated at 4 and 24 hai in the resistant NIL, respectively (Table 1). The Arabidopsis orthologs for these set of genes were obtained and finally 224 Arabidopsis genes were ultimately submitted to the TF2Network server. For a given set of functionally related genes, this resource outputted a list of regulators (TFs) and target genes based on the results from hypergeometric tests of motifs (transcription factor binding sites) in the input genes. Approximately 624 genes, including TFs, were obtained in the regulatory network, and 89 and 114 of these genes were mapped to the magenta and salmon modules, respectively. The network was filtered to retain only the TFs included in the magenta module and their target genes in the salmon module that were exclusively up-regulated in the resistant NIL. The final network shown in Figure 5 indicates that OsbZIP45 is a centrally important TF in the magenta module and has connections with all the genes in the salmon module.

A significant motif enrichment (score $q$-value of 0.026 based on BenjaminiHochberg correction for multiple hypotheses testing (Benjamini et al., 1995) was obtained for the bZIP motifs on the target genes. This gene and its Arabidopsis ortholog GBF3 have been reported to be involved in drought tolerance (Liu et al., (2015). We also found that 
several TFs interact with other TFs, such as bHLH148 with MYB24 (score of 0.73) (Table Supplementary 6). To further confirm the probable regulatory roles of the subnetwork of OsbZIP45 in rice, we performed an in silico promoter analysis in which $1 \mathrm{~kb}$ upstream sequences of the target genes (61) of OsbZIP45 were extracted and submitted to PlantPAN (v3.0) (Chow et al., 2019) for "Gene Group Analysis" for co-occurrence of TF-binding sites (TFBS). The list of TFBS with high confidence scores $(>90)$ is provided in Table Supplementary 7. The presence of the TF-binding sites in various genes, such as bZIP, MYB, bHLH, and WRKY, provides support to the regulatory subnetwork in the rice resistant genotype. A recent study showed that MYB cooperates with BHLH proteins in regulatory networks that control phenylpropanoid biosynthetic pathways and epidermal cell differentiation and patterning in Arabidopsis (Ramegowda et al., 2017). Here, we observed a network of regulatory interactions among plant defense and lipid and secondary mechanisms that notably integrate with the phenylpropanoid pathway (Table Supplementary 4). We also identified 61 TF-target genes associated with OsbZIP45 TFs and most of these TF-target genes are regulatory genes involved in calcium signaling, posttranscriptional modifications (PTMs) and transcription (e.g., WRKYs 7, 15, 28, and 94). Defense-related genes include the PR and redox-related genes. A small percentage of genes were involved in metabolic pathways such as lignin biosynthesis, lipid metabolism and hormone metabolism (brassinosteroid and ethylene biosynthesis).

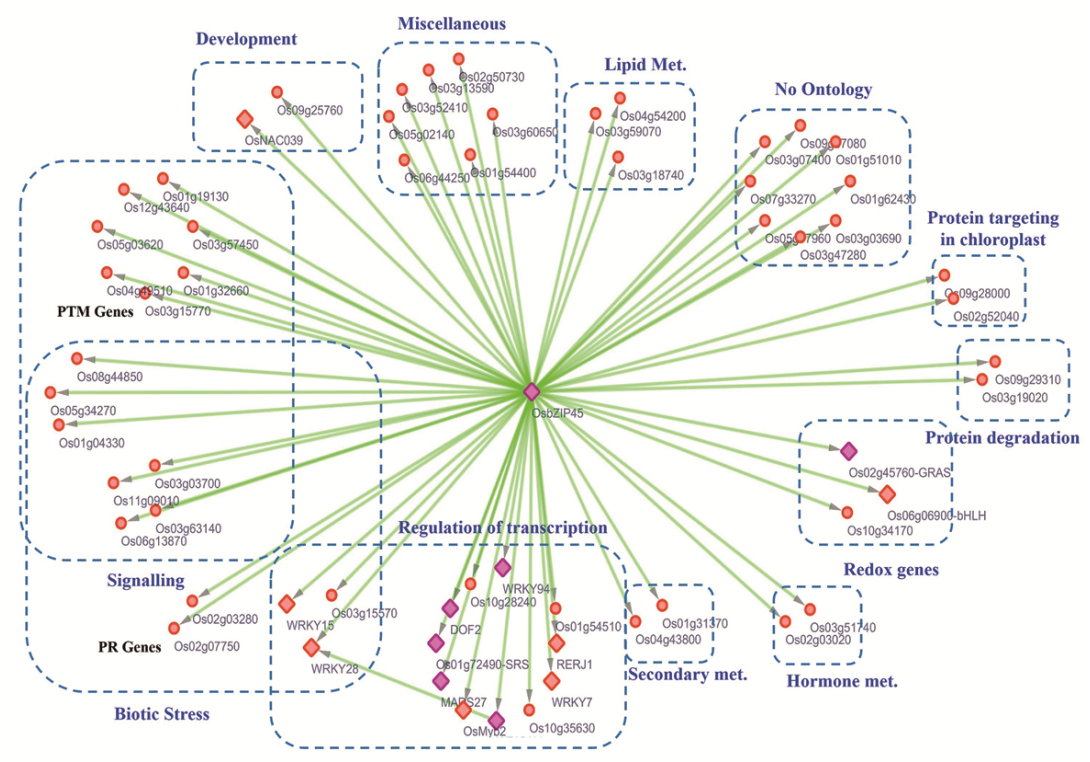

Figure 5. TF-target gene regulatory network extracted from TF2Network. The circular nodes indicate genes, whereas the diamond nodes indicate TFs. The salmon and magenta colors represent the respective modules. The edges represent regulatory interactions. The nodes were clustered manually, using MapMan functional annotations. The genes in the salmon module are exclusively up-regulated at 24 hai in the resistant NIL. 


\section{DISCUSSION}

This study provides a new perspective for evaluating large-scale transcription data using a co-expression network in combination with PPI analysis, and we applied this strategy to the analysis of gene expression datasets from rice in response to $M$. oryzae infection during an incompatible reaction. Our results demonstrated that this integrative network analysis was able to identify genes involved in rice resistance in response to $M$. oryzae. Specifically, the analysis revealed that the occurrence of a set of hub genes specifically co-expressed at the very early stage of infection was critical to the outcome of rice blast resistance because these genes were exclusively up-regulated at 4 hai in the resistant NIL. Consistent with the extensive plant-fungal exchanges observed throughout the first 24 hours, the resistant NIL responded successfully to $M$. oryzae infection through a complex network involving a potent signaling pathway, phytohormones, secondary metabolism and secondary messengers such as calcium and stress receptors. Protein kinase/phosphatase cascades and lipid metabolism are other important components of this network.

The protein-protein interactions occurring during appressorium formation at 24 hai might play essential roles, particularly in signaling mediated by receptor kinases, TF recruitment, hormone signaling mediated by brassinosteroids and jasmonates, and lipid metabolism, bHLH which suggests that the resistant NIL established a strong network to mount a defense against $M$. oryzae infection. Additionally, genes directly involved in plantpathogen interactions were also directly associated. The distinct roles of these interactions in the defense responses of rice to $M$. oryzae infection might be strongly related to the interaction partners of the identified hubs. For example, a protein kinase and a ZIM domain containing protein (a JAZ protein) were found to have 18 and 17 partners (Table Supplementary 4), respectively, which is consistent with the important role of JA and receptor-like kinases in signaling during the early stages of the response of the resistant NIL to M. oryzae infection. Additionally, as part of the JA-mediated responses, the bHLH TF activated different sets of genes and, thereby established a strong network with the jasmonate ZIM domain. The bHLH TFs encode a MYC-related transcriptional activator with a typical DNA binding domain of a basic helix-loop-helix leucine zipper motif (Pireyre and Brown, 2015) and have been shown to play key roles in JA signaling responses to pathogens. In our study, bHLH established a strong network with the jasmonate ZIM domain (JAZ protein). This interaction has been well demonstrated by several researchers. For example, in Arabidopsis under stress-free growth conditions, JA responses are restrained by a jasmonate-Zim-domain (JAZ) repressor, which directly targets the basic helix-loop-helix TF AtMYC2 (Gimenez-Ibanez and Solano, 2013). Exogenous jasmonates or herbivory stimulate binding of the F-box protein CORONATINE-INSENSITIVE 1 (COI1) to jasmonate ZIM domain (JAZ) proteins. The COI1-JAZ complex are targeted for degradation by the $26 \mathrm{~S}$ proteasome (Chini et al., 2007).

AtMYC2 is then released to activate the expression of JA responsive genes to accomplish its function. It was recently revealed that AtMYC3 and AtMYC4 are targets of JAZ repressors that act additively with AtMYC2 in the activation of the jasmonate response to Pseudomonas syringae in Arabidopsis (Fernandez-Calvo, et al., 2011). In rice, the overexpression of OsMYC2 induces the up-regulation of early JA-responsive genes and strong resistance to bacterial blight caused by Xanthomonas oryzae (Uji et al., 2016). 
Interestingly, Seo et al., (2011) observed a similar interaction mechanism after the overexpression of OsbHLH148 in rice. They proposed the existence of an OsbHLH148OsJAZ-OsCOI1 complex in a jasmonate signaling pathway and this interaction enhanced drought tolerance in rice. These findings might help to uncover whether MYCs are JAZinteracting TFs regulate JA responses after M. oryzae infection, but further experiments are needed to confirm this hypothesis.

Additionally, our data showed strong interactions between OsMYB4 and other components of the phenylpropanoid pathway, which represent a diverse family of secondary metabolites that originate from phenylalanine. The first three steps in the synthesis of phenylpropanoid-derived compounds are catalyzed by phenylalanine ammonia lyase (PAL), cinnamate 4-hydroxylase $(\mathrm{CHH})$, and p-coumaroyl coenzyme A ligase $(4 \mathrm{CL})$, and these reactions are collectively referred to as the general phenylpropanoid pathway (GPP). GPP products serve as precursors for phenylpropanoid-derived compounds. Our study revealed that Os4CL5 interacts with OsPAL3 and OsMYB4, which interact with OsbHLH148 partnered with OsUCL29, and the resulting complex interacts with OsPAL3 (Table Supplementary 4). In many cases, bHLHs are able to physically interact with different MYBs to achieve their function (Feller et al., 2011). This interaction might suggest that cross-talk among these genes or pathways are needed for the rapid production of lignin to strengthen the plant cell wall and consequently enhance rice disease resistance. It is well known that lignin biosynthesis extensively contributes to responses to a variety of biotic and abiotic stresses. When reinforced, the cell wall serves as a physical barrier to fungus penetration. The recognition of pathogens by pattern recognition receptors and/or resistance proteins activates defense signals involving salicylic acid (SA), JA and ethylene, and this activation results in plant defense responses that include the biosynthesis of lignin reinforce cell walls and to prevent the entrance of pathogens. Additionally, the cell wall is a reservoir of antimicrobial compounds that are released during cell wall degradation. Based on these results, we can conclude that the resistant NIL fights against the fungus in an unspecific or general manner to prevent or reduce damage and energy costs. In other words, PTI is extensively activated in this line. In contrast, even though these basal defenses are also activated in the susceptible line, the activation of these responses was not sufficient to completely stop the pathogen. The resistance shown by the resistant NIL also required ETI, which involves the detection of specific avirulence effector (AVR) genes that are mediated by NBS-LRR proteins.

Also noteworthy is the interaction of NB-ARC/LRR with several genes, especially OsWRKY70 and OsWRKY24. Inoue et al., 2013 have demonstrated that the disease resistance protein PANICLE BLAST 1 (Pb1), which encodes a CC-NB-LRR protein, physically interacted with the transcription factor WRKY45 regulating the rice defense responses against $M$. oryzae via the salicylic acid signaling pathway. Using subcellular localization, bimolecular fluorescence complementation and co-immunoprecipitation experiments, Chakraborty et al. (2018) found that chickpea defense response to Fusarium oxysporum f. sp. ciceri race1 (Focl) depends on CC-NB-ARC/LRR interaction with WRKY64 transcription factor. WRKY64 physically interacts with CC-NB-ARC/LRR, which phosphorylates WRKY64 and phosphorylated WRKY64 binds to EDS1 (ENHANCED DISEASE SUSCEPTIBILITY1 (EDS1) promoter and stimulates its transcription, generating a strong immune response against $F o c 1$. Based on these results, we might speculate that NB-ARC/LRR-OsWRKY70 or NB-ARC/LRR-OsWRKY24 might be 
important players in rice resistance to M. oryzae. Furthermore, the role of these interactions in rice resistance to $M$. oryzae and their relation with Pi5 remains to be experimentally elucidated.

Interestingly, several MYB/bHLH complexes have been described. Different $\mathrm{MYB} / \mathrm{bHLH}$ complexes regulate distinct cellular processes such as cell wall synthesis, cell death, the circadian clock, responses to abiotic and biotic stress, hormone signaling, and the biosynthesis of specialized metabolites (Pireyre and Burow, 2015). The MYB-and -bHLH interaction in the regulation of phenylpropanoid metabolism related to plant disease is unknown. However, previous studies of this interaction in Eudicots indicated that it regulates the synthesis of anthocyanin phenylpropanoid-derived compounds. In this case, MYB is activated by stressed leaves and forms a transcriptional complex with bHLH and the WD-repeat protein, and the resulting complex activated the transcription of its targets, including the genes encoding dihydroflavonol 4-reductase (DFR), bHLH2, R2R3-MYB repressor, and R3-MYB repressor. Sugars and hormones can also modulate anthocyanin biosynthesis via coordinated regulation of the R2R3-MYB/bHLH/WD40 (MBW) complex. In contrast, this complex is not required for anthocyanin pigmentation in maize, which appears to be an independent regulated process (Ramegowda et al., 2017). Transgenic rice plants expressing maize anthocyanin genes exhibit increased blast resistance (Gandikota et al., 2001), but the role of this pathway in conferring disease resistance in rice has not yet been characterized.

The discovery of TF-targeted genes has typically been used for analyzing the complete set of interactions identified in many organisms at a genome-wide scale. In our study, instead of using this approach at a genome-wide scale, we focused on a small set of transcriptional changes induced by $M$. oryzae during an incompatible reaction with rice. The combination of an in silico TF regulatory interactions with a promoter analysis in rice indicated a role for $O s b Z I P 45$ as a driver of complex gene expression changes that resulted in the rice blast resistance. OsbZIP45 might act as a TF hub because it binds to the promoter of many genes. TF hubs have a higher tendency to be essential for the function of an organism (Deplanke et al., 2006). In multicellular organisms, gene expression is precisely regulated in both in time and space (Ramegowda et al., 2017) and this fact was exemplified in our data set. The analysis of the regulatory network constructed from our data showed that, although OsbZIP45 was activated at 4 hai, it became highly connected to its target genes only at 24 hai. We speculate that OsbZIP45 might be involved in delaying the activation of the several sets of TF-target genes that should be useful at 24 hai and thus, adjusting to the new metabolic scenario imposed by the appressorium penetration. We suggest the existence of a time lag between the initiation of OsbZIP45 and the activation of its targets. Based on these computational models, we may consider OsbZIP45 as a regulatory hub that orchestrates the rice defense response induced by $M$. oryzae infection at 4 hai and probably regulating the expression of TF-target genes at 24 hai. This hypothesis is also consistent with the fact that the response time and transcription level are vital parameters of gene regulation.

Overall, our results showed that transcription factors identified here may act interactively rather than alone. This finding is important when it is necessary to define strategies to develop transgenic plants expressing rice blast resistance. Possible strategies to produce plants with increased disease resistance using transcription factors was discussed by Tripathi et al., (2016). For example, to reduce pleiotropic effects caused when using TFs 
as transgenes, they suggested restricting transgene expression to infection sites by using pathogen-inducible promoters. They also proposed the manipulation of TFs that function as activators or repressors as a key mechanism for the activation of plant defenses using CRISPR and RNAi to produce transgenic lines with altered TF expression with improved disease resistance.

\section{CONCLUSIONS}

We examined the potential network defense responses in rice to M. oryzae infection during an incompatible reaction. The genes and TFs identified are of high biological relevance, and the results revealed a putative role for uncharacterized genes that might play a role in the rice-pathogen interaction. The network analysis revealed that the interaction among TFs, lipid metabolism and secondary metabolism coupled with the phenylpropanoid pathway appears to be a key defense mechanism in rice against $M$. oryzae that leads to various adaptive responses, including deposition in the resistant NIL. We suggest that the OsMYB/bHLH regulatory network and the OsMYC/OsbHLH/JAZ complex, which have not been reported to be involved in rice resistance to $M$. oryzae, play a regulatory role in this pathosystem. Together, the responses generated by these interactions appear to play an important role in the defense of rice against M. oryzae. Additionally, OsbZIP45 may act as an integrator of multiple signals and a coordinator of diverse cellular pathways to control the defense responses in rice. We suggeste that the temporal and spatial regulation of OsbZIP45 activation in the resistant NIL at 4 hai is an important aspect of the signaling activity observed in this NIL at 24 hai. However, the above-described biological processes need to be validated to determine the properties revealed by our network analyses.

\section{ACKNOWLEDGMENTS}

We thank Embrapa (the Brazilian Agricultural Research Agency) for funding and support.

\section{CONFLICTS OF INTEREST}

The authors declare no conflict of interest.

\section{REFERENCES}

Anders S and Huber W (2010). Differential expression analysis for sequence count data. Genome Biol. 11: R106.

Anders S, Pyl PT and Huber W (2015). HTSeq--a Python framework to work with high-throughput sequencing data. Bioinformatics. 31: 166-169.

Aoki K, Ogata Y and Shibata D (2007). Approaches for extracting practical information from gene co-expression networks in plant biology. Plant Cell Physiol. 48: 381-90.

Bagnaresi P, Biselli C, Orru L, Urso S, et al. (2012). Comparative transcriptome profiling of the early response to Magnaporthe oryzae in durable resistant vs susceptible rice (Oryza sativa L.) genotypes. PLoS One. 7: e51609.

Barabasi AL and Oltvai ZN (2004). Network biology: understanding the cell's functional organization. Nat. Rev. Genet. 5: 101-113.

Benjamini Y and Hochberg Y (1995). Controlling the false discovery rate: a practical and powerful approach to multiple testing. J R Stat Soc Ser B Methodol. 57: 289-300.

Bolger AM, Lohse M and Usadel B (2014). Trimmomatic: a flexible trimmer for Illumina sequence data. Bioinformatics. 30: $2114-2120$ 
Bundo M and Coca M (2016). Enhancing blast disease resistance by overexpression of the calcium-dependent protein kinase OsCPK4 in rice. Plant Biotechnol. J. 14: 1357-67.

Chakraborty J, Priya P, Dastidar SG and Das S (2018). Physical interaction between nuclear accumulated CC-NB-ARCLRR protein and WRKY64 promotes EDS1 dependent Fusarium wilt resistance in chickpea. Plant Sci. 276: 111133.

Chen X, Shang J, Chen D, Lei C, et al. (2006). A B-lectin receptor kinase gene conferring rice blast resistance. Plant J. 46: 794-804.

Chini A, Fonseca S, Fernandez G, Adie B, et al. (2007). The JAZ family of repressors is the missing link in jasmonate signalling. Nature. 448: 666-71.

Chow CN, Lee TY, Hung YC, Li GZ, et al. (2019). PlantPAN3.0: a new and updated resource for reconstructing transcriptional regulatory networks from ChIP-seq experiments in plants. Nucleic Acids Res. 47: D1155-1163.

Deplancke B, Mukhopadhyay A, Ao W, Elewa AM, et al. (2006). A gene-centered C. elegans protein-DNA interaction network. Cell. 125: 1193-1205.

Duan L, Liu H, Li X, Xiao J, et al. (2014). Multiple phytohormones and phytoalexins are involved in disease resistance to Magnaporthe oryzae invaded from roots in rice. Physiol. Plantarum. 152: 486-500.

Feller A, Machemer K, Braun EL and Grotewold E (2011). Evolutionary and comparative analysis of MYB and bHLH plant transcription factors. Plant J. 66: 94-116.

Fernandez-Calvo P, Chini A, Fernandez-Barbero G, Chico JM, et al. (2011). The Arabidopsis bHLH transcription factors MYC3 and MYC4 are targets of JAZ repressors and act additively with MYC2 in the activation of jasmonate responses. Plant Cell. 23: 701-715.

Filippi MC and Prabhu AS (2001). Phenotypic virulence analysis of Pyricularia grisea isolates from Brazilian upland rice cultivars. Pesq. Agropec. Bras. 36: 27-35.

Gandikota M, Kochko A, Chen L, Ithal N, et al. (2001). Development of transgenic rice plants expressing maize anthocyanin genes and increased blast resistance. Mol. Breed. 7: 73-83.

Garcia-Alcalde F, Okonechnikov K, Carbonell J, Cruz LM, et al. (2012). Qualimap: evaluating next-generation sequencing alignment data. Bioinformatics. 28: 2678-2679.

Gimenez-Ibanez S and Solano R (2013). Nuclear jasmonate and salicylate signaling and cross talkin defense against pathogens. Front Plant Sci. 4: 72.

Hong LI and Ziding Z (2016). Systems understanding of plant-pathogen interactions through genome-wide proteinprotein interaction networks. Front Agr. Sci. Eng. 3: 102-112.

Inoue $\mathrm{H}$, Hayashi N, Matsushita, A, Xinqiong L, et al. (2013). Blast resistance of CC-NB-LRR protein Pb1 is mediated by WRKY45 through protein-protein interaction. Proc. Natl. Acad. Sci. U. S. A. 110: 9577-9582.

Kawahara Y, de la Bastide M, Hamilton JP, Kanamori H, et al. (2013). Improvement of the Oryza sativa Nipponbare reference genome using next generation sequence and optical map data. Rice (N Y). 6: 4.

Koizumi S (2007). Durability of resistance to rice blast disease. In: Fukuta Y, Cruz CMV, Kabayashi N, editors. JIRCAS Working Report no 53: a differential system for blast resistance for stable rice production environment. Tsukuba: JIRCAS. p. 1-15.

Kulkarni SR, Vaneechoutte D, Van de Velde J and Vandepoele K (2017). TF2Network: predicting transcription factor regulators and gene regulatory networks in Arabidopsis using publicly available binding site information. Nucleic Acids Res. 46: e31.

Langfelder P and Horvath S (2008). WGCNA: an R package for weighted correlation network analysis. $B M C$ Bioinformatics. 9: 559.

Langfelder P, Mischel PS and Horvath S (2013). When is hub gene selection better than standard meta-analysis? PLoS One 8: e61505.

Lee SK, Song MY, Seo YS, Kim H, et al. (2009). Rice Pi5-mediated resistance to Magnaporthe oryzae requires the presence of two coiled-coil-nucleotide-binding-leucine rich repeat genes. Genetics. 181: 1627-1638.

Liu J, Osbourn A and Ma P (2015). MYB Transcription Factors as Regulators of Phenylpropanoid Metabolism in Plants. Mol. Plant. 8: 689-708.

Li H, Handsaker B, Wysoker A, Fennell T, et al. (2009). The sequence alignment/map format and SAMtools. Bioinformatics. 25: 2078-2079.

Lv S, Wang Z, Yang X, Guo L, et al. (2016). Transcriptional Profiling of Rice Treated with MoHrip1 Reveal the Function of Protein Elicitor in Enhancement of Disease Resistance and Plant Growth. Front Plant Sci.7: 1818.

Moore JW, Loake GJ and Spoel SH (2011). Transcription dynamics in plant immunity. Plant Cell. 23: 2809-2820.

Nalley L, Tsiboe F, Durand-Morat A, Shew A, et al. (2016). Economic and environmental impact of rice blast pathogen (Magnaporthe oryzae) alleviation in the United States. PLoS One. 11: e0167295.

Onaga G, Wydra K, Koopmann B, Chebotarov D, et al. (2017). High temperature effects on Pi54 conferred resistance to Magnaporthe oryzae in two genetic backgrounds of Oryza sativa. J. Plant Physiol. 212: 80-93.

Pireyre M and Burow M (2015). Regulation of MYB and bHLH transcription factors: a glance at the protein level. Mol. Plant. 8: 378-388.

Ramegowda V, Gill US, Sivalingam PN, Gupta A, et al. (2017). GBF3 transcription factor imparts drought tolerance in Arabidopsis thaliana. Sci. Rep. 7: 9148.

Genetics and Molecular Research 19 (3): gmr18579

CFUNPEC-RP www.funpecrp.com.br 
Ribot C, Hirsch J, Balzergue S, Tharreau D, et al. (2008). Susceptibility of rice to the blast fungus, Magnaporthe grisea. J. Plant Physiol. 165: 114-124.

Seo JS, Joo J, Kim MJ, Kim YK, et al. (2011). OsbHLH148, a basic helix-loop-helix protein, interacts with OsJAZ proteins in a jasmonate signaling pathway leading to drought tolerance in rice. Plant J. 65: 907-921.

Sircar S and Parekh N (2015). Functional characterization of drought-responsive modules and genes in Oryza sativa: a network-based approach. Front. Genet. 6: 256.

Szklarczyk D, Franceschini A, Wyder S, Forslund K, et al. (2015). STRING v10: protein-protein interaction networks, integrated over the tree of life. Nucleic Acids Res. 43: D447-452.

Talbot NJ (2003). On the trail of a cereal killer: exploring the biology of Magnaporthe grisea. Annu. Rev. Microbiol. 57: 177-202.

Thimm O, Blasing O, Gibon Y, Nagel A, et al. (2004). MAPMAN: a user-driven tool to display genomics data sets onto diagrams of metabolic pathways and other biological processes. Plant J. 37: 914-939.

Tsuda K and Somssich IE (2015). Transcriptional networks in plant immunity. New Phytol. 206: 932-947.

Tsunematsu H, Yanoria MJT, Ebron LA, Hayashi N, et al. (2000). Development of monogenic lines of rice for blast resistance. Breed Sci. 50: 229-234.

Tripathi P, Galla A, Rabara RC and Rushton PJ (2016). Transcription Factors that Regulate Defence Responses and Their Use in Increasing Disease Resistance. In Plant Pathogen Resistance Biotechnology, D.B. Collinge (Ed.). doi:10.1002/9781118867716.ch6.

Uji Y, Taniguchi S, Tamaoki D, Shishido H, et al. (2016). Overexpression of OsMYC2 results in the up-regulation of early JA-responsive genes and bacterial blight resistance in rice. Plant Cell Physiol. 57: 1814-1827.

Voigt A, Nowick K and Almaas E (2017). A composite network of conserved and tissue specific gene interactions reveals possible genetic interactions in glioma. PLoS Comput. Biol. 13: e1005739.

Wei T, Ou B, Li J, Zhao Y, et al. (2013). Transcriptional Profiling of Rice Early Response to Magnaporthe oryzae Identified OsWRKYs as Important Regulators in Rice Blast Resistance. PLoS One. 8(3): e59720.

Whitfield ML, Sherlock G, Saldanha AJ, Murray JI et al. (2002). Identification of genes periodically expressed in the human cell cycle and their expression in tumors. Mol. Biol. Cell. 13: 1977-2000. 